\title{
Three-dimensional assessment of maxillary changes associated with bone anchored maxillary protraction
}

\author{
Tung Nguyen ${ }^{a}$, Lucia Cevidanes ${ }^{a}$, Marie A. Cornelis ${ }^{b}$, Gavin Heymann ${ }^{c}$, Leonardo K. de \\ Paula $^{d}$, and Hugo De Clerck $^{e}$
}

aAssistant professor, Department of Orthodontics, School of Dentistry, University of North Carolina, Chapel Hill. bAssistant professor, Department of Orthodontics, School of Dentistry, University of Geneva, Geneva, Switzerland. 'Private practice, Durham, NC. ${ }^{\mathrm{d}}$ Research assistant, Department of Orthodontics, School of Dentistry, University of North Carolina, Chapel Hill.

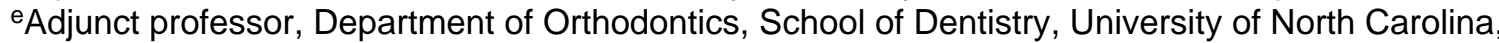
Chapel Hill.

\begin{abstract}
Introduction-Bone-anchored maxillary protraction has been shown to be an effective treatment modality for the correction of Class III malocclusions. The purpose of this study was to evaluate 3-dimensional changes in the maxilla, the surrounding hard and soft tissues, and the circummaxillary sutures after bone-anchored maxillary protraction treatment.

Methods-Twenty-five consecutive skeletal Class III patients between the ages of 9 and 13 years (mean, $11.10 \pm 1.1$ years) were treated with Class III intermaxillary elastics and bilateral miniplates ( 2 in the infrazygomatic crests of the maxilla and 2 in the anterior mandible). Conebeam computed tomographs were taken before initial loading and 1 year out. Three-dimensional models were generated from the tomographs, registered on the anterior cranial base, superimposed, and analyzed by using color maps.
\end{abstract}

Results-The maxilla showed a mean forward displacement of $3.7 \mathrm{~mm}$, and the zygomas and the maxillary incisors came forward 3.7 and $4.3 \mathrm{~mm}$, respectively.

Conclusions-This treatment approach produced significant orthopedic changes in the maxilla and the zygomas in growing Class III patients.

Maxillary hypoplasia is frequently found in patients with Class III malocclusion.

Conventional treatments involve the use of a protraction facemask to advance the maxilla. However, such appliances often have unwanted side effects including maxillary incisor proclination and clockwise rotation of the mandible. ${ }^{1-3}$ New treatment methods with skeletal anchorage in the maxillary buttress have been developed to minimize dentoalveolar compensations. ${ }^{4-6}$ These protocols, however, still need a facemask. De Clerck et al ${ }^{7}$ suggested the use of Class III elastics between miniplate skeletal anchorage in both jaws (bone-anchored maxillary protraction). Preliminary studies based on conventional 2dimensional (2D) cephalometric data after active therapy show significantly favorable results from bone-anchored maxillary protraction compared with control and facemask groups. 8,9

Copyright $\odot 2011$ by the American Association of Orthodontists.

Reprint requests to: Tung Nguyen, Department of Orthodontics, UNC School of Dentistry, CB\#7450, Chapel Hill, NC 27599-7450; nguyentu@dentistry.unc.edu..

The authors report no commercial, proprietary, or financial interest in the products or companies described in this article. 
The precise response to protraction of the maxilla with bone-anchored maxillary protraction, including remodeling and adaptation of the adjacent bones, sutures, and soft tissues, has yet to be clarified. Furthermore, the envelope of correction that is possible with bone-anchored maxillary protraction needs to be established. These morphologic changes and their clinical relevance cannot be well addressed with 2D imaging methods alone. Advances in 3dimensional (3D) imaging of facial structures provide an alternative tool to analyze skeletal changes. The development and validation of a method for 3D longitudinal assessments by using surface registration on the anterior cranial base as a reference could identify the location and nature of the orthopedic effects after bone-anchored maxillary protraction. ${ }^{10}$

In a pilot study on 3D assessments of treatment outcomes of bone-anchored maxillary protraction, Heymann et $\mathrm{al}^{11}$ reported marked variability in responses to the therapy. Our study expands these preliminary 3D findings to a larger sample, to evaluate overall facial changes relative to the cranial base at the end of active treatment with bone-anchored maxillary protraction. The purpose of this study was to evaluate in 3 dimensions the growth and treatment effects of the bone-anchored maxillary protraction protocol on the maxillary dentition, the midface, and the adjacent soft tissues of consecutively treated patients.

\section{MATERIAL AND METHODS}

This prospective sample consisted of 25 consecutively treated patients (13 girls, 12 boys) with dentoskeletal Class III malocclusion. All patients were treated by 2 operators (H.D.C. and M.A.C.) with the bone-anchored maxillary protraction technique.

At the initial observation (T1), all patients had Class III malocclusion in the mixed or permanent dentition characterized by a Wits appraisal of $-1 \mathrm{~mm}$ or less (mean, $-4.8 \pm 2.8$ $\mathrm{mm}$ ), anterior crossbite or incisor end-to-end relationship, and Class III molar relationship. All patients were of white ancestry, with a prepubertal stage of skeletal maturity according to the cervical vertebral maturation method (CS1-CS3). ${ }^{12}$ Twenty-one of the 25 patients were still prepubertal at the end of treatment (T2), and 4 patients were at CS4. The mean ages for the bone-anchored maxillary protraction sample were $11.9 \pm 1.8$ years at $\mathrm{T} 1$ and $13.1 \pm 1.7$ years at $\mathrm{T} 2$. The mean duration of the $\mathrm{T} 1$ to $\mathrm{T} 2$ interval was $1.2 \pm 1.0$ years. Institutional review board approval for the study was obtained from the University of North Carolina.

Each patient had miniplates placed on the left and right infrazygomatic crests of the maxillary buttress and between the mandibular left and right lateral incisors and canine (Fig 1). Small mucoperiosteal flaps were elevated, and the modified miniplates (Bollard, TitaLink, Brussels, Belgium) were secured to the bone by 2 (mandiblular) or 3 (maxillary) screws (diameter, $2.3 \mathrm{~mm}$; length, $5 \mathrm{~mm}$ ). ${ }^{7}$ The extensions of the plates perforated the attached gingiva near the mucogingival junction (Fig 2). Three weeks after surgery, the miniplates were loaded. Class III elastics applied an initial force of $100 \mathrm{~g}$ on each side, increased to $150 \mathrm{~g}$ after 1 month of traction and to $250 \mathrm{~g}$ after 3 months. The patients were asked to replace the elastics at least once a day and to wear those 24 hours per day. In 14 patients, after 2 to 3 months of intermaxillary traction, a removable bite plate was inserted in the maxillary arch to eliminate occlusal interference.

Cone-beam computed tomography (CBCT) scans were taken at $\mathrm{T} 1$ and $\mathrm{T} 2$ by using an iCat machine (Imaging Sciences International, Hatfield, Pa) with a $16 \times 22$-cm field of view. Virtual 3D surface models were constructed from the CBCT images with a voxel dimension of $0.5 \times 0.5 \times 0.5 \mathrm{~mm}$. Construction of 3D surface models of the anatomic structures of interest and the 3D graphic renderings were done by using the ITK-SNAP (open-source software; www.itksnap.org). 
The $\mathrm{T} 1$ and $\mathrm{T} 2$ images were registered by using the anterior cranial fossa as a reference. A fully automated voxel-wise rigid registration method was performed with IMAGINE (opensource software; http://www.ia.unc.edu/dev/download/imagine/index.htm). This method, developed by Cevidanes et al, ${ }^{13}$ masks anatomic structures altered by treatment or growth to prevent observer-dependent reliance on subjectively defined anatomic landmarks. In this study, the initial and final 3D models were registered on anterior cranial fossa structures, specifically the endocranial surfaces of the cribiform plate region of the ethmoid bone and the frontal bone. These regions were chosen because of their early completion of growth. The software compares 2 images by using the intensity gray scale for each voxel of the T1 and $\mathrm{T} 2$ images.

After the registration step, all reoriented virtual models were superimposed to quantitatively evaluate the greatest surface displacement by using the CMF application software (developed at the M. E. Müller Institute for Surgical Technology and Biomechanics, University of Bern, Bern, Switzerland, under the funding of the Co-Me network; http://come.ch). This tool calculates thousands of color-coded point-to-point comparisons (surface distances in millimeters) between the 3D models, so that the difference between 2 surfaces at any location can be quantified.

For quantitative assessment of the changes between the 3D surface models, the isoline tool allows the user to define a surface-distance value to be expressed as a contour line (isoline) that corresponds to regions having a surface distance equal to or greater than the defined value. The isoline tool was used to quantitatively measure the greatest displacements between points in the 3D surface models for the maxillary incisors, the maxilla, the right and left zygomas, the upper lip, and the soft-tissue nose (Fig 3). Positive values indicated anterior displacement, and negative values posterior displacement. For the maxillary incisor, the maximum surface distance was measured at the incisor's most labial surface. The maxillary region was defined as the anterior surface of the maxilla between the canines. The right and left zygomas were defined as the surfaces adjacent and superior to the bone anchors. The soft-tissue nose region was de-fined as the tip of the nose. The upper lip was defined as the area below the nose between the lip commissures.

\section{Statistical analysis}

The largest displacements between $\mathrm{T} 1$ and $\mathrm{T} 2$ were computed at each anatomic region of interest. The error of the method as determined in previous studies showed excellent reliability. ${ }^{10}$ Descriptive statistics were used to describe the sample percentiles, means, standard deviations, and ranges. Pearson correlation coefficients were used to assess the associations between changes at each anatomic region. The levels of significance were set at 0.05 for $P$ values and 0.5 for $r$ values.

\section{RESULTS}

Table I summarizes the descriptive statistics for the skeletal and soft-tissue changes observed from $\mathrm{T} 1$ to $\mathrm{T} 2$ for the 25 consecutively treated bone-anchored maxillary protraction patients. The maxilla showed a mean displacement of $3.73 \mathrm{~mm}$, whereas the maxillary incisors and the right and left zygomas advanced 4.27, 3.60, and $3.76 \mathrm{~mm}$, respectively. Although all subjects had anterior displacements of the skeletal and dental structures of interest, there were considerable variations in the magnitudes (Fig 4). Skeletal changes between $\mathrm{T} 1$ and $\mathrm{T} 2$ are displayed as color maps or semitransparencies (Figs 5 and $6)$.

The soft-tissue upper lip advanced $3.98 \mathrm{~mm}$, and the nose translated forward $3.82 \mathrm{~mm}$. Softtissue displacements also showed a wide range of variations. A color map and mesh 
superimpositions showing these changes between $\mathrm{T} 1$ and $\mathrm{T} 2$ for 9 subjects are given in Figures 7 and 8, respectively.

Pearson analysis showed significant correlations between the displacement of the maxillary incisors, the maxilla, the zygomas, and the soft-tissue upper lip (Table II). There was a significant correlation among the regions except for the soft-tissue nose with the left and right zygomas.

\section{DISCUSSION}

The purposes of this study were to evaluate an adequate sample of consecutively treated Class III patients by using the bone-anchored maxillary protraction protocol and to report the 3D changes to the skeletal, dental, and soft tissues of the midface region. Twodimensional studies of maxillary protraction have limitations, since the landmarks evaluated are often midline structures or 2D projections of 3D structures. Furthermore, bilateral structures cannot be evaluated individually and are susceptible to measurement errors if the patient's head is slightly rotated during film capture. CBCT scans and evolving 3D imaging softwares have resolved many of these issues and given a more global view of the surface changes, not only in the region of interest, but also in the neighboring structures.

Bone-anchored maxillary protraction treatment has been shown to produce significant orthopedic changes compared with untreated Class III subjects and facemask or rapid maxillary expansion therapy. Two-dimensional cephalometric data showed $4 \mathrm{~mm}$ of maxillary improvement with bone-anchored maxillary protraction treatment, measured at Apoint, when compared with the untreated controls. In addition, bone-anchored maxillary protraction produced 2.3 to $3 \mathrm{~mm}$ more of maxillary protraction compared with facemask or rapid maxillary expansion treatment. ${ }^{9}$ Although $3 \mathrm{D}$ untreated normative data are not available for direct comparison with the bone-anchored maxillary protraction treatment group, indirect comparisons can be made with the 2D data. Mean values for maxillary incisor and maxillary displacement in our 3D study correlated well with the 2D findings. Three-dimensional color maps and semi-transparency superimpositions showed no significant proclination of the maxillary incisors with bone-anchored maxillary protraction (Figs 5 and 6). This is in contrast to a meta-analysis of the effectiveness of facemask therapy by Kim et al, ${ }^{14}$ which identified increased labial inclination of the maxillary incisor and decreased mandibular incisor inclination with conventional protraction facemask therapy.

Every subject in this prospective sample of consecutively treated patients had forward displacement in the zygomatic and infraorbital regions, with mean changes of 3.6 to $3.8 \mathrm{~mm}$. This finding was similar to the $2 \mathrm{D}$ cephalometric data from this sample, which reported a 3.5-mm anterior displacement of orbitale in the bone-anchored maxillary protraction group. However, 3D color maps clearly locate the areas of bone remodeling and displacement. In this sample, there was a statistically significant correlation $(P<0.0001)$ between the magnitude of displacement of the left and right zygomas, the maxilla, and the maxillary incisors, suggesting that the midface was displaced anteriorly as a unit.

Three-dimensional data on untreated controls and patients treated by other techniques for maxillary protraction are not currently available. For this reason, comparison of results with other techniques refers to $2 \mathrm{D}$ cephalometric findings. The amount of maxillary protraction with bone-anchored maxillary protraction is more significant when compared with $0.6 \mathrm{~mm}$ of forward growth of orbitale in untreated Class III controls over a similar time period. ${ }^{8}$ Previously reported findings of infraorbital changes after facemask therapy are controversial. Nartallo-Turley and Turley ${ }^{15}$ reported significant advancements of orbitale (2 $\mathrm{mm})$ and the key ridge $(1 \mathrm{~mm})$, whereas Pangrazio-Kulbersh et $\mathrm{al}^{16}$ found no effect on the 
displacement of orbitale in young patients treated with facemask protraction. The mean age in the facemask group of Nartallo-Turley and Turley was 7.3 years compared with 8.9 years in the group of Pangrazio-Kulbersh et al and 11.9 years in our bone-anchored maxillary protraction group. Baccetti et al ${ }^{17}$ also indicated greater maxillary orthopedic effects of facemasks in the early mixed dentition. Future 3D studies with facemask therapy are needed to evaluate and compare the effectiveness in producing orthopedic changes at the infraorbital and zygomatic regions.

The 3D color maps (Fig 5) show that bone-anchored maxillary protraction patients had uniform anterior displacements of the maxilla and the zygoma. Our 2D cephalometric study showed minimal counterclockwise rotation of the maxilla with bone-anchored maxillary protraction treatment. ${ }^{8}$ These findings contradict the hypothetical definition of Teuscher ${ }^{18}$ of the position of the maxillary center of resistance in the biomechanics of bone displacement. If the center of resistance were located at the maxillary buttress, the line of force of the bone-anchored maxillary protraction Class III elastics to this center of resistance would result in a moment of force and a marked counterclockwise rotation of the maxilla. Our study showed minimal rotation of the maxilla, suggesting that the center of resistance of the mid-face is probably located posterior and inferior to that defined by Teuscher.

Another interesting finding was the opening of the circummaxillary sutures that was evident in a number of subjects. Forward displacement of the maxilla in this study was observed without disarticulation of the facial sutures as advocated by Liou ${ }^{19}$ and Wang et al. ${ }^{20}$ Contrary to facemask protocols, the bone-anchored maxillary protraction protocol was not preceded by a rapid palatal expansion. Future investigations are needed to assess whether the circummaxillary suture opens because of the direct application of forces to the maxillary bone in the bone-anchored maxillary protraction protocol.

Displacement of the maxilla is greatly influenced by the resistance against movement at the circummaxillary sutures. Also, the complexity of the suture interdigitations, which increase with age, affects the orthopedic outcome. ${ }^{21}$ From animal studies, it is known that the suture surface and the complexity of interdigitations are higher in the zygomaticomaxillary suture than in the zygomaticotemporal and zygomaticofrontal sutures. The transverse palatine suture has shown the highest opening of all circummaxillary sutures after protraction of the maxilla in primates. ${ }^{22,23}$ These findings were confirmed by opening of the sutures as observed on the CBCT scans at $\mathrm{T} 2$ of the bone-anchored maxillary protraction sample (Fig 9). Although many of our patients exhibited this pattern of sutural opening at T2, it was not seen in all of them. This is to be expected, since CBCT scans at T2 can only capture this phenomenon during periods of rapid orthopedic distraction, and before bone deposition and maturation have occurred. Melsen ${ }^{21}$ suggested that the palatine bone should be considered as a buffer between the facial skeleton and the cranial base. This might explain the difference in the amounts and the directions of displacement of the anterior and posterior nasal spines as shown by the thin plate spline analysis of this sample. ${ }^{24}$ The opening or stimulus of growth at the maxillary sutures is a gradual process when light forces are applied, but, in some subjects, the sutural opening was clearly visible at T2. The high potential of adaptation in the transverse palatine, zygomaticotemporal, and zygomaticofrontal sutures might explain why the maxilla, the zygomas, and the maxillary incisors moved forward as 1 unit.

The 3D changes in the midface in this study were at the end of active therapy, and assessments of mandibular compensation and adaptations to the bone-anchored maxillary protraction protocol are needed. Overall treatment changes after fixed appliances and the pubertal growth spurt will be needed to assess the long-term stability of correction with the 
bone-anchored maxillary protraction protocol and the percentage of these patients who might still require surgical correction after completion of facial growth.

\section{CONCLUSIONS}

This 3D evaluation of the therapeutic effects of the bone-anchored maxillary protraction protocol in Class III growing patients showed significant maxillary and zygomatic protraction with hardly any skeletal rotational changes or dental compensation of the maxillary incisors. The soft-tissue changes were also significant and comparable, although evident only at the level of the upper lip.

\section{Acknowledgments}

We thank Camilla J. Tulloch and Hong Tu Zhu for their assistance on the project and the National Institute of Dental and Craniofacial Research and American Association of Orthodontists Foundation for their support.

Supported by the National Institute of Dental and Craniofacial Research (NIDCR) number DE005215 and the American Association of Orthodontists Foundation.

\section{REFERENCES}

1. Baik HS. Clinical results of the maxillary protraction in Korean children. Am J Orthod Dentofacial Orthop. 1995; 108:583-92. [PubMed: 7503035]

2. Kapust AJ, Sinclair PM, Turley PK. Cephalometric effects of face mask/expansion therapy in Class III children: a comparison of three age groups. Am J Orthod Dentofacial Orthop. 1998; 113:204-12. [PubMed: 9484212]

3. Takada K, Petdachai S, Sakuda M. Changes in dentofacial morphology in skeletal Class III children treated by a modified maxillary protraction headgear and a chin cup: a longitudinal cephalometric appraisal. Eur J Orthod. 1993; 15:211-21. [PubMed: 8339762]

4. Singer SL, Henry PJ, Rosenberg I. Osseointegrated implants as an adjunct to facemask therapy: a case report. Angle Orthod. 2000; 70:253-62. [PubMed: 10926436]

5. Enacar A, Giray B, Pehlivanoglu M, Iplikcioglu H. Facemask therapy with rigid anchorage in a patient with maxillary hypoplasia and severe oligodontia. Am J Orthod Dentofacial Orthop. 2003; 123:571-7. [PubMed: 12750679]

6. Kircelli BH, Pektas ZO. Midfacial protraction with skeletally anchored face mask therapy: a novel approach and preliminary results. Am J Orthod Dentofacial Orthop. 2008; 133:440-9. [PubMed: 18331946]

7. De Clerck HJ, Cornelis MA, Cevidanes LH, Heymann GC, Tulloch CJ. Orthopedic traction of the maxilla with miniplates: a new perspective for treatment of midface deficiency. J Oral Maxillofac Surg. 2009; 67:2123-9. [PubMed: 19761906]

8. De Clerck H, Cevidanes L, Baccetti T. Dentofacial effects of bone-anchored maxillary protraction: a controlled study on consecutively treated Class III patients. Am J Orthod Dentofacial Orthop. 2010; 138:577-81. [PubMed: 21055597]

9. Cevidanes L, Baccetti T, Franchi L, McNamara JA Jr, De Clerck H. Comparison of two protocols for maxillary protraction: bone anchors versus face mask with rapid maxillary expansion. Angle Orthod. 2010; 80:799-806. [PubMed: 20578848]

10. Cevidanes LH, Heymann G, Cornelis MA, De Clerck HJ, Tulloch JF. Superimposition of 3dimensional cone-beam computed tomography models of growing patients. Am J Orthod Dentofacial Orthop. 2009; 136:94-9. [PubMed: 19577154]

11. Heymann GC, Cevidanes L, Cornelis M, De Clerck HJ, Tulloch JF. Three-dimensional analysis of maxillary protraction with inter-maxillary elastics to miniplates. Am J Orthod Dentofacial Orthop. 2010; 137:274-84. [PubMed: 20152686]

12. Baccetti T, Franchi L, McNamara JA Jr. The cervical vertebral maturation (CVM) method for the assessment of optimal treatment timing in dentofacial orthopedics. Semin Orthod. 2005; 11:11929. 
13. Cevidanes LH, Styner MA, Proffit WR. Image analysis and superim-position of 3-dimensional cone-beam computed tomography models. Am J Orthod Dentofacial Orthop. 2006; 129:611-8. [PubMed: 16679201]

14. Kim JH, Viana MA, Graber TM, Omerza FF, BeGole EA. The effectiveness of protraction face mask therapy: a meta-analysis. Am J Orthod Dentofacial Orthop. 1999; 115:675-85. [PubMed: 10358251]

15. Nartallo-Turley PE, Turley PK. Cephalometric effects of combined palatal expansion and facemask therapy on Class III malocclusion. Angle Orthod. 1998; 68:217-24. [PubMed: 9622758]

16. Pangrazio-Kulbersh V, Berger J, Kersten G. Effects of protraction mechanics on the midface. Am J Orthod Dentofacial Orthop. 1998; 114:484-91. [PubMed: 9810043]

17. Baccetti T, McGill JS, Franchi L, McNamara JA Jr, Tollaro I. Skeletal effects of early treatment of Class III malocclusion with maxillary expansion and face-mask therapy. Am J Orthod Dentofacial Orthop. 1998; 113:333-43. [PubMed: 9517727]

18. Teuscher U. An appraisal of growth and reaction to extraoral anchorage: simulation of orthodonticorthopedic results. Am J Orthod. 1986; 89:113-21. [PubMed: 3456205]

19. Liou EJ. Effective maxillary orthopedic protraction for growing Class III patients: a clinical application simulates distraction osteo-genesis. Prog Orthod. 2005; 6:154-71. [PubMed: 16276426]

20. Wang YC, Chang PM, Liou EJ. Opening of circumaxillary sutures by alternate rapid maxillary expansions and constrictions. Angle Orthod. 2009; 79:230-4. [PubMed: 19216603]

21. Melsen B. Palatal growth studied on human autopsy material. A histologic microradiographic study. Am J Orthod. 1975; 68:42-54. [PubMed: 1056143]

22. Jackson GW, Kokich VG, Shapiro PA. Experimental and postexperimental response to anteriorly directed extraoral force in young Macaca nemestrina. Am J Orthod. 1979; 75:318-33. [PubMed: 107808]

23. Zhao N, Xu Y, Chen Y, Xu Y, Han X, Wang L. Effects of Class III magnetic orthopedic forces on the craniofacial sutures of rhesus monkeys. Am J Orthod Dentofacial Orthop. 2008; 133:401-9. [PubMed: 18331940]

24. Baccetti T, De Clerck HJ, Cevidanes LHS, Franchi L. Morphometric analysis of treatment effects of bone-anchored maxillary protraction in growing Class III patients. Eur J Orthod. 2011; 33:1215. [PubMed: 21187527] 


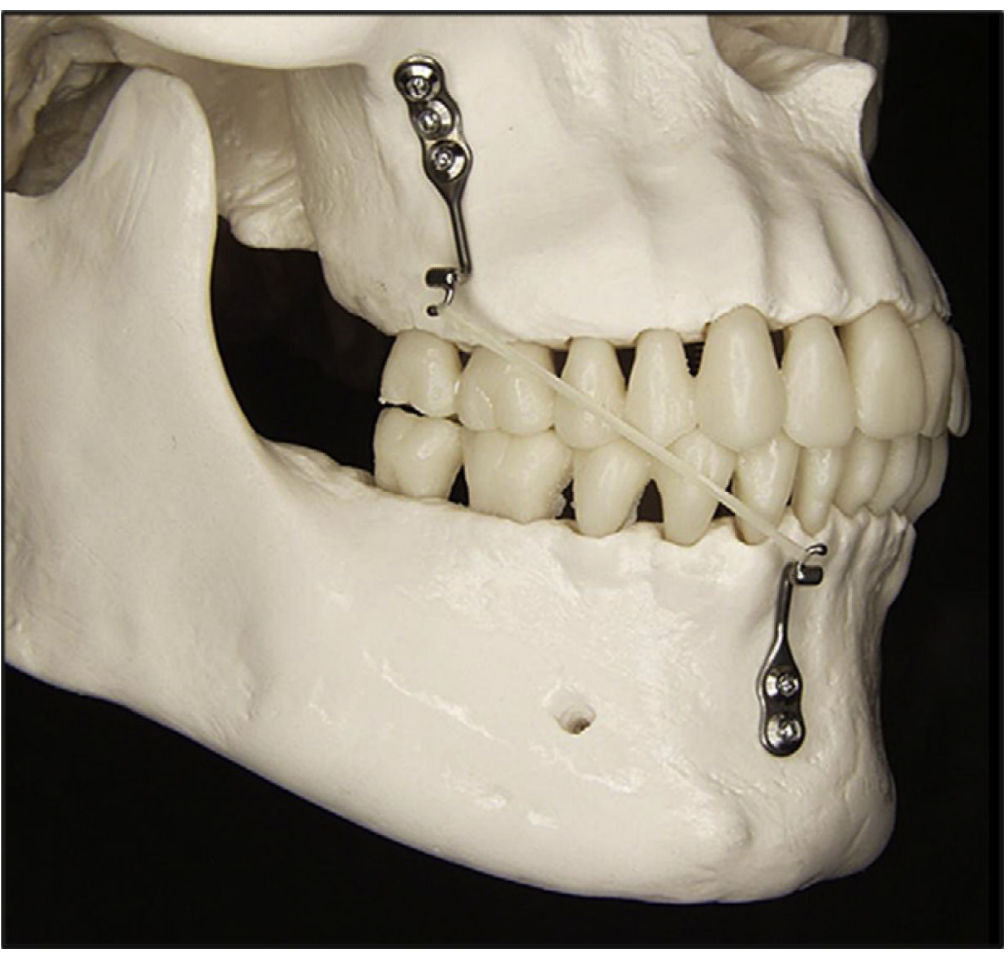

Fig 1.

Skull illustrating the anatomic locations of the bone anchors. 


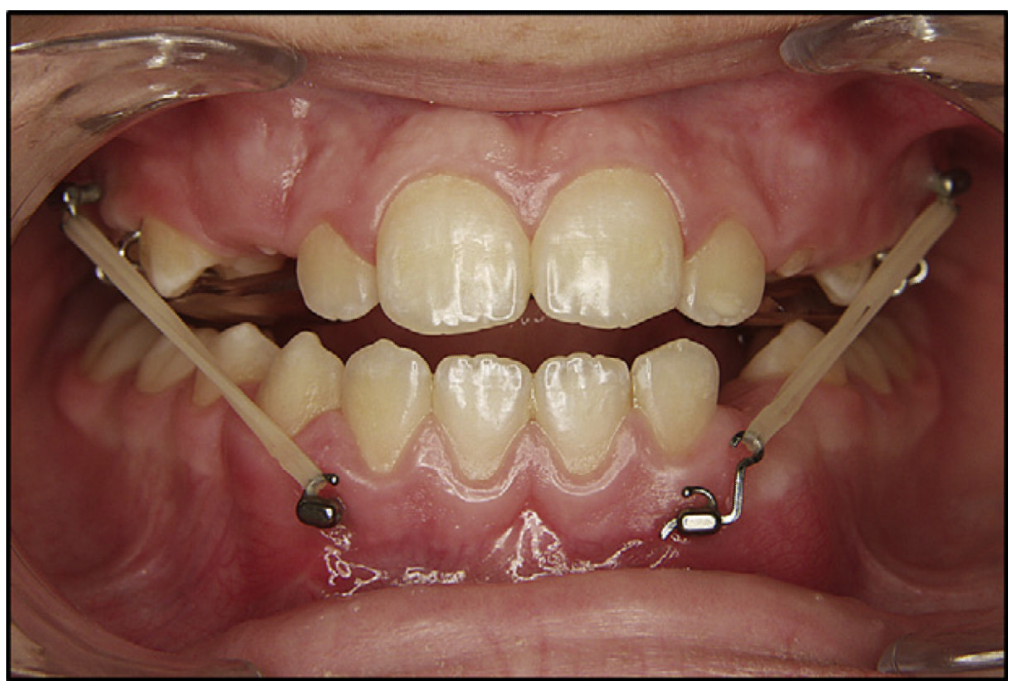

Fig 2.

Intermaxillary elastics to anchors with a bite plate retainer to assist with bite opening. 


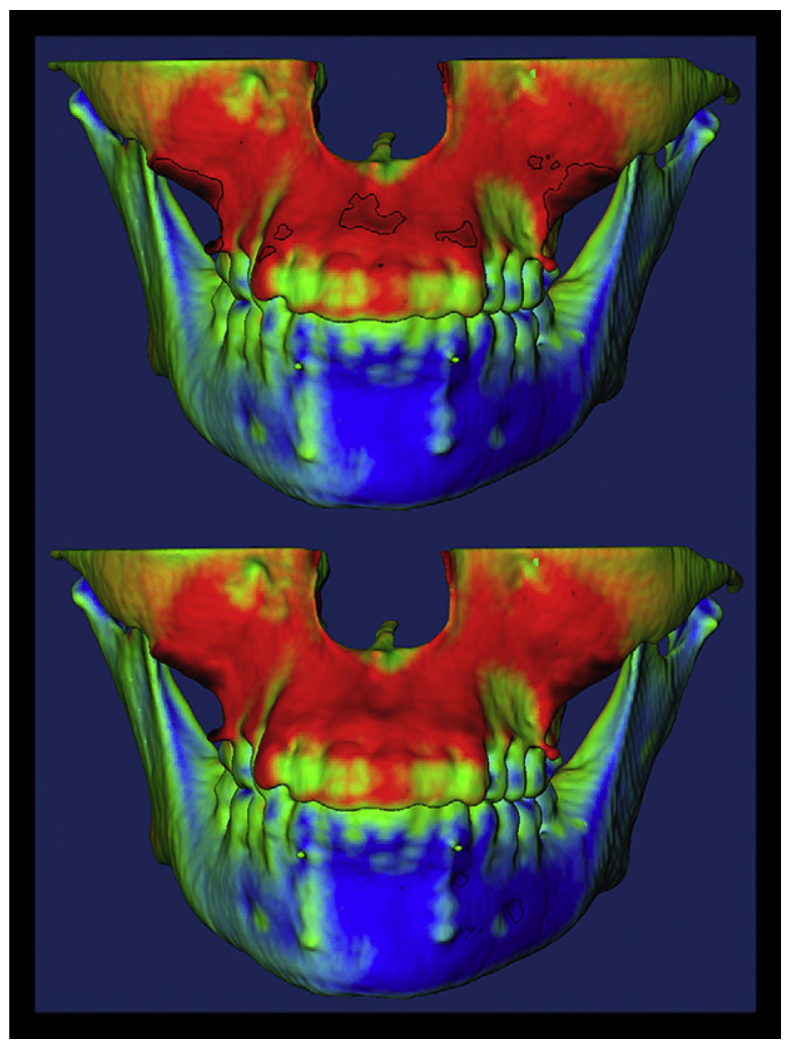

Fig 3.

The isolines, displayed as a black circle or a dot, show the regions with the greatest displacement (positive in red; negative in blue) between the T1 and T2 superimpositions. These isolines measure the closest distance between the superimpositions. The top image shows the isolines representing the regions of greatest outward displacement in the maxilla and zygomas, and the bottom image illustrates the regions with maximum inward displacement. 


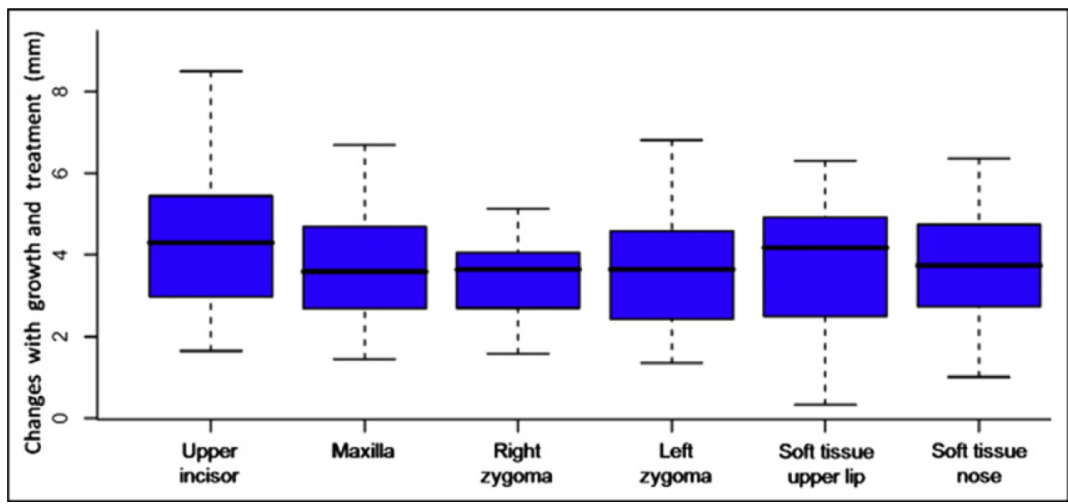

Fig 4.

Box plots of the growth and response to treatment at each anatomic region. 

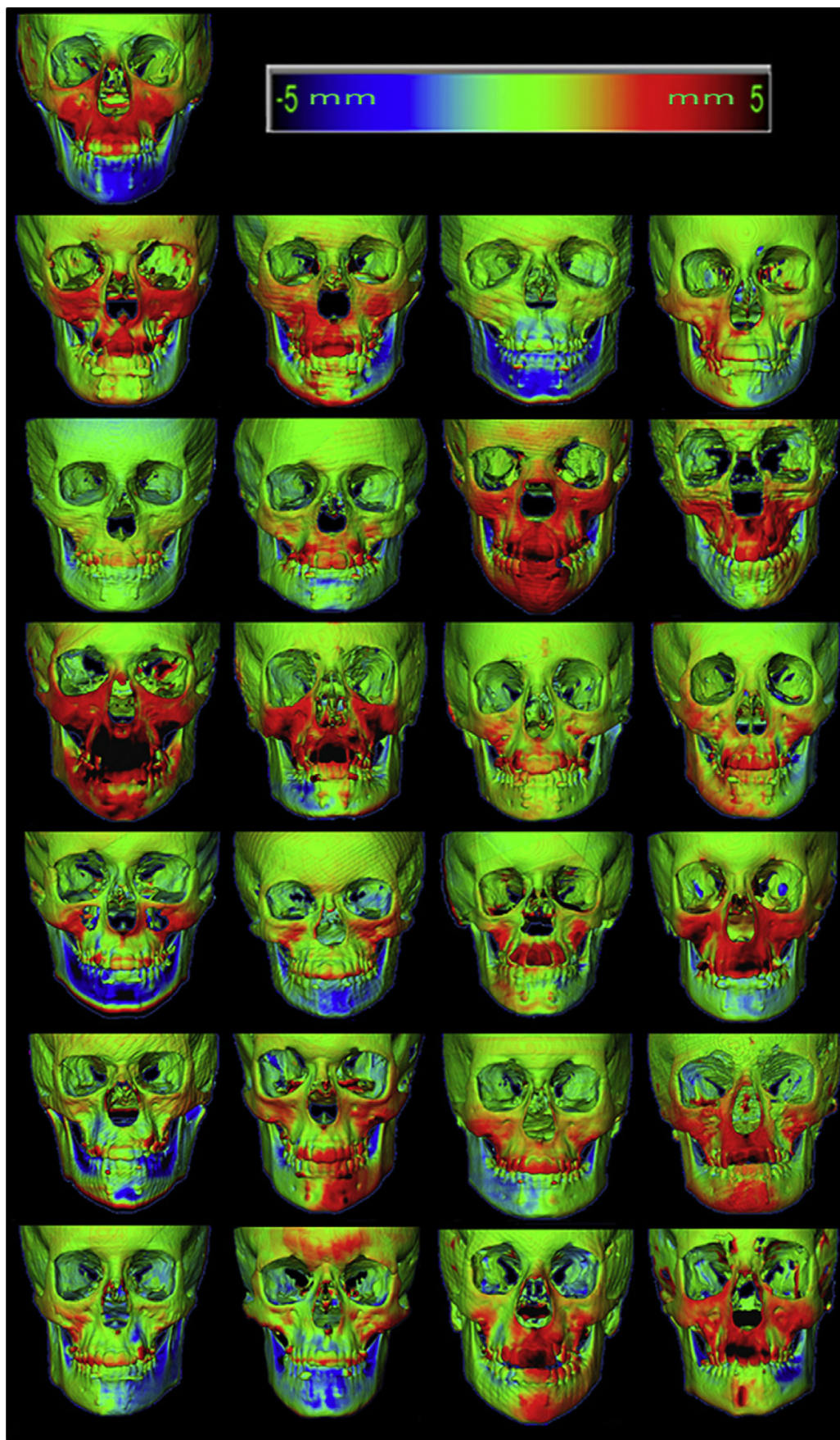

Fig 5.

Three-dimensional skeletal color maps of superimpositions of T2 over T1 registered at the anterior cranial base with a scale of -5 to $+5 \mathrm{~mm}$. Red represents outward displace of T2 in relation to T1; blue represents inward displacement of $\mathrm{T} 2$ in relation to $\mathrm{T} 1$. 


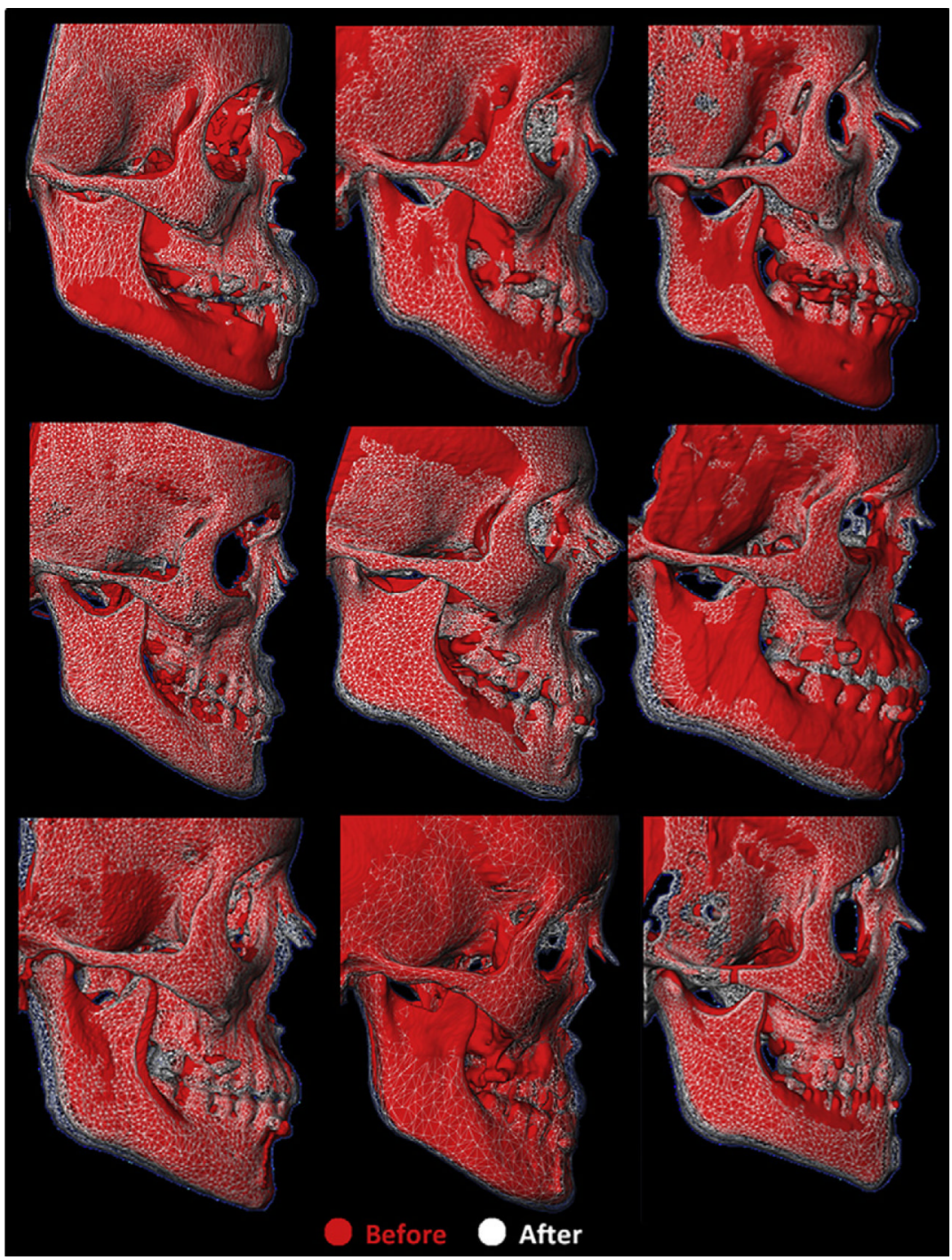

Fig 6.

Lateral views of the skeletal semitransparency superimpositions for 9 patients. T1 models are represented by solid red; superimposed T2 models are shown as semitransparent white mesh. 


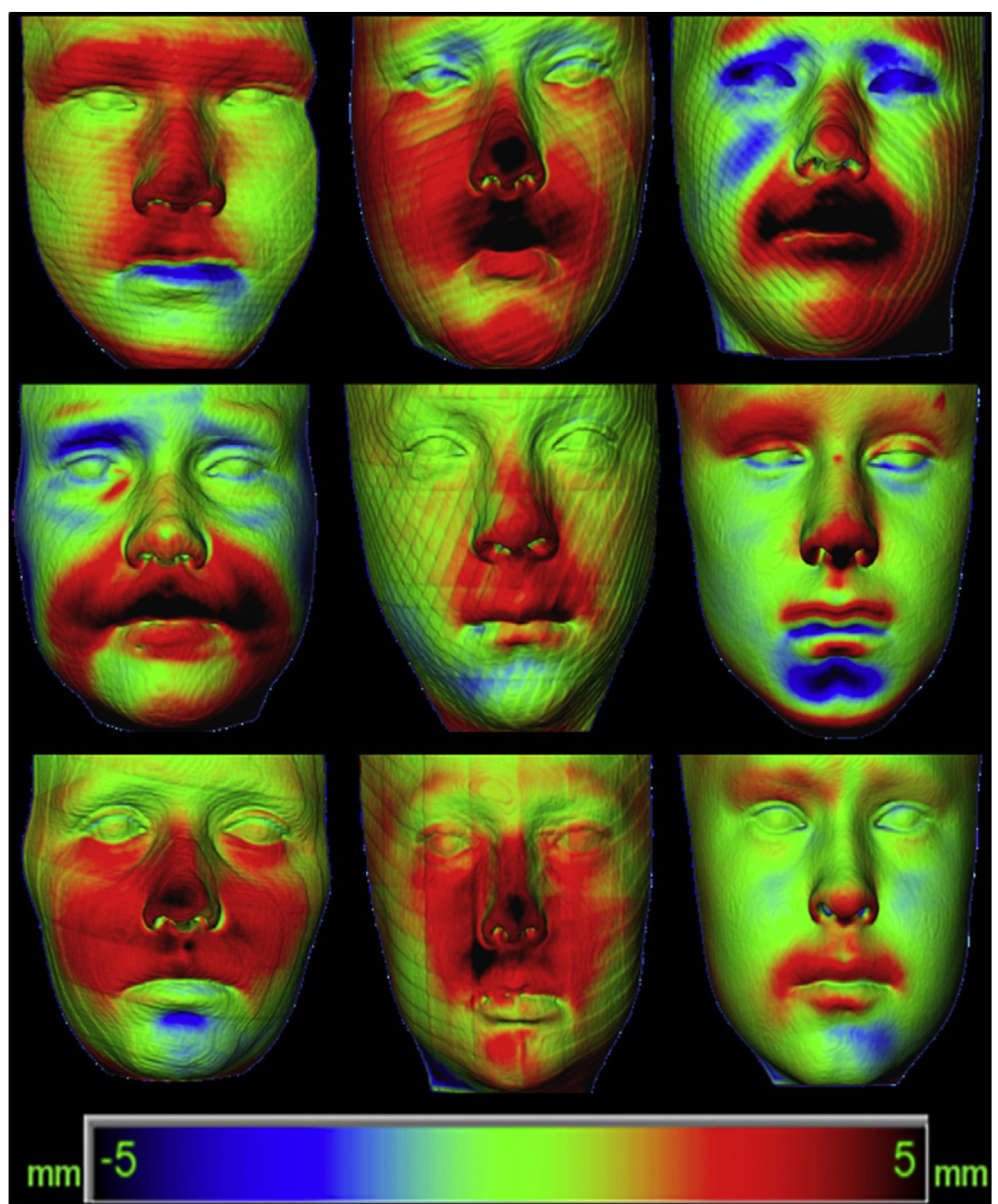

Fig 7.

Frontal views of 3D soft-tissue color maps of superimpositions for 9 patients showing a large range of responses. 


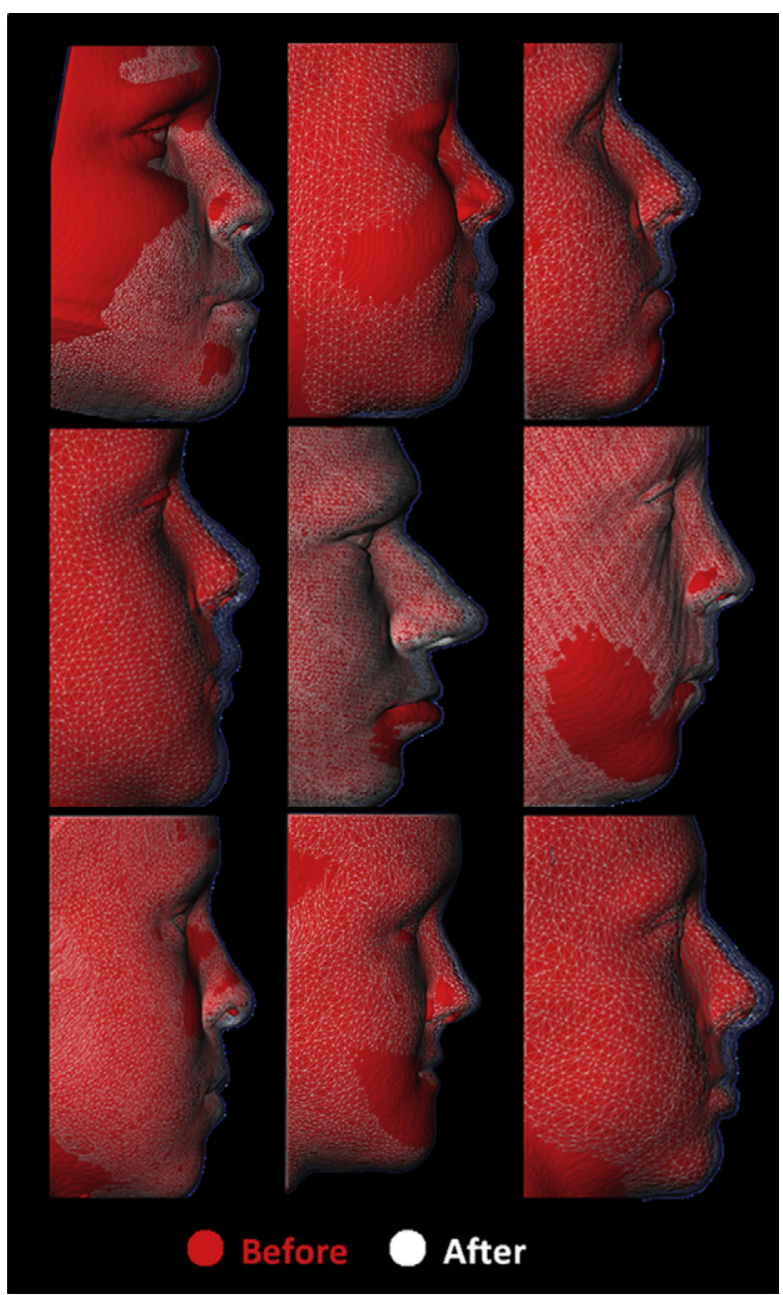

Fig 8.

Lateral views of the soft-tissue semitransparency superimpositions for 9 patients. Note the range of soft-tissue responses from 1 patient to another. 


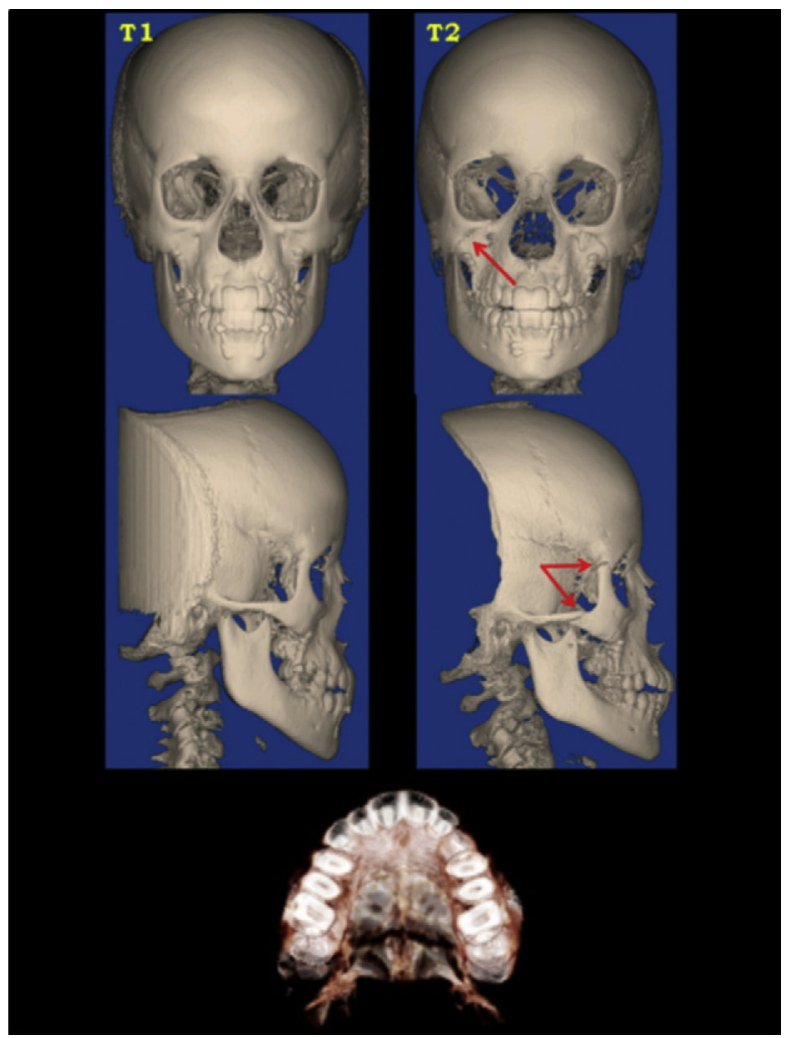

Fig 9.

Distraction of circummaxillary sutures at T2 (red arrows). Note the opening of the zygomaticofrontal, zygomaticotemporal, zygomaticomaxillary, and transverse palatine sutures. 


\section{Table I}

Mean values, standard deviations, and ranges of maxillary protraction changes in millimeters at each anatomic region relative to the cranial base superimposition

\begin{tabular}{lcccccc}
\hline & Maxillary incisor & Maxilla & Right zygoma & Left zygoma & Soft-tissue upper lip & Soft-tissue nose \\
Mean and SD & $4.27 \pm 1.66$ & $3.73 \pm 1.66$ & $3.60 \pm 1.32$ & $3.76 \pm 1.53$ & $3.98 \pm 1.82$ & $3.82 \pm 1.36$ \\
Range & $1.65-8.50$ & $1.45-8.5$ & $1.58-6.86$ & $1.35-7.81$ & $0.33-9.32$ & $1.01-6.36$ \\
\hline
\end{tabular}


Table II

Pearson correlation coefficients for growth and treatment response changes between all anatomic regions of interest

\begin{tabular}{lcccccc}
\hline Anatomic region & Maxillary incisor & Maxilla & Right zygoma & Left zygoma & Soft-tissue upper lip & Soft-tissue nose \\
Maxillary incisor & & $0.92^{*}$ & $0.87^{*}$ & $0.87^{*}$ & $0.83^{*}$ & $0.50^{*}$ \\
Maxilla & $<0.0001^{*}$ & & $0.85^{*}$ & $0.83^{*}$ & $0.82^{*}$ & $0.53^{*}$ \\
Right zygoma & $<0.0001^{*}$ & $<0.0001^{*}$ & & $0.91^{*}$ & $0.73^{*}$ & 0.30 \\
Left zygoma & $<0.0001^{*}$ & $<0.0001^{*}$ & $<0.0001^{*}$ & & $0.75^{*}$ & 0.38 \\
Soft-tissue upper lip & $<0.0001^{*}$ & $<0.0001^{*}$ & $<0.0001^{*}$ & $<0.0001{ }^{*}$ & & $0.66^{*}$ \\
Soft-tissue nose & $0.01^{*}$ & $0.01{ }^{*}$ & 0.15 & 0.06 & $<0.001{ }^{*}$ & \\
\hline
\end{tabular}

The upper right section of the table shows $r$ values, and the lower left section of the table shows the $P$ values. Statistical signifcance values were set at 0.05 for $P$ and 0.5 for $r$.

* Signifcant values. 